\title{
A IMPORTÂNCIA DO OBSERVATÓRIO DE ATIVIDADES INDUSTRIAIS VIS-À-VIS TENDÊNCIAS EM CIÊNCIA, TECNOLOGIA E INOVAÇÃO
}

\author{
Adelaide Maria de Souza Antunes* e Ana Carolina Silva Mangueira \\ Escola de Química, Universidade Federal do Rio de Janeiro, 21949-900 Rio de Janeiro - RJ
}

\begin{abstract}
THE IMPORTANCE OF THE OBSERVATORY OF INDUSTRIAL ACTIVITIES AND TRENDS IN SCIENCE, TECHNOLOGY AND INNOVATION. Given that innovation is fundamental for competitiveness, and that it is intrinsically linked to knowledge, it has become essential to foster effective interaction between the Brazilian productive sector and universities and R\&D centers. This interface should be created by means of the model used in developed countries: Observatory of Industrial Activities and Trends in Science, Technology and Innovation. The present work describes its main functions, using examples of activities undertaken by the Chemical Industry Information System of the Federal University of Rio de Janeiro related to production systems of primary and high-tech industries, examining the diffusion of knowledge to decision-makers.
\end{abstract}

Keywords: observatory of industrial activities; trends in Science, Technology and Innovation; market and technology mining.

\section{OBSERVATÓRIO DE ATIVIDADES INDUSTRIAIS E DE TENDÊNCIAS EM CIÊNCIA, TECNOLOGIA E INOVAÇÃO (C,T\&I)}

A motivação principal da modelagem do Observatório de Atividades Industriais e de Tendências em C,T\&I está na necessidade de estruturação do Sistema Setorial de Inovação, que vem ao encontro do perfil da Lei de Inovação promulgada em dezembro/2004, que dispõe sobre incentivos à pesquisa científica e tecnológica e à inovação, e dá outras providências.

Para alavancar a competitividade da indústria, é necessário que o Estado reconheça a importância do conhecimento para desenvolver a capacidade inovadora local.

O valor dos produtos e serviços cresce como função dos conhecimentos neles incorporados. É importante, portanto, assinalar que a cooperação universidade-empresa é um importante instrumento de interação, que deve ser realizada de forma a maximizar os benefícios para seus objetivos e, conseqüentemente, para a sociedade como um todo. Embora com objetivos bastante diferenciados, universidades, institutos e empresas são parte de um sistema.

As reflexões e ações na direção de parcerias entre universidades e empresas datam do século XIX, demonstrando uma busca contínua por melhores padrões da qualidade de vida e maior acumulação de riquezas, bens e serviços.

O ideal é que haja um organismo de interface capaz de conhecer a linguagem empresarial, seu comportamento e expectativas e, ao mesmo tempo, conhecer a qualidade dos conhecimentos disponíveis, o potencial dos pesquisadores e equipe.

Cabe destacar que, dentre as diretrizes do Ministério da Ciência e Tecnologia (MCT), se verifica dentre as prioridades de ação, o apoio à coordenação que implemente projetos cooperativos e de interesse comum, na direção da inovação e competitividade.

Assim sendo, o modelo do Observatório deve ter os seguintes papéis/funções:

- facilitador da interação entre as Universidades, o Setor Produtivo e o Poder Público;

- identificar demandas empresariais e oportunidades de novas frentes de trabalho para o desenvolvimento econômico e social do País;

\footnotetext{
*e-mail: adelaide@eq.ufrj.br
}

- considerar a inovação como um processo dinâmico e cumulativo; - operar sistematicamente por meio de ações de curto, médio e longo prazos;

- conscientizar e mobilizar o conhecimento para o desenvolvimento sustentável através de Fóruns Temáticos e/ou setoriais e,

- acompanhar o processo dinâmico da inovação tecnológica.

Tendo em vista as funções apresentadas anteriormente, o modelo proposto para o Observatório é apresentado no Esquema 1:

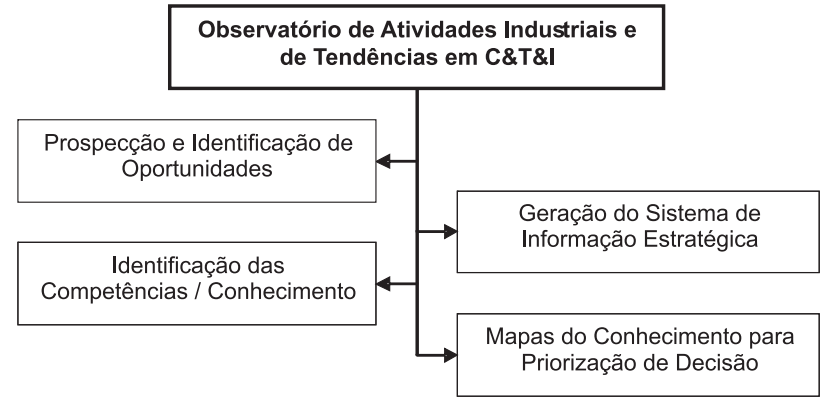

Esquema 1. Modelo da estrutura do Observatório de Atividades Industriais e de Tendências em $C, T \& I$

A estrutura do Observatório será detalhada, com as respectivas funções propostas no modelo:

Prospecção e Identificação de Oportunidades - a modelagem do Sistema de Prospecção leva em conta aspectos de Tecnologias portadoras de Futuro; de Mercado; Sociais e, de Análise de oportunidades de parcerias universidade/empresa (prospecção tecnológica, monitoramento das competências nas universidades e centros de P\&D do País vis-a-vis as vocações, sua capacidade produtiva e empreendedora).

Geração do Sistema de Informação Estratégica - envolve a localização de nichos de oportunidades, com avaliação e atualização de novas vocações vis-a-vis novos desafios e gargalos.

Identificação das Competências/Conhecimento - envolve o monitoramento: das competências científicas; dos setores industriais e de serviços; das vocações das regiões e dos Arranjos Produtivos Locais e, de produto e serviços.

Mapas do Conhecimento para Priorização da Decisão - utilização de ferramentas de tratamento automático da informação 
para geração de mapas de conhecimento, visando o acompanhamento tanto das mudanças tecnológicas, como dos impactos econômico-sociais.

\section{O SISTEMA DE INFORMAÇÃO SOBRE A INDÚSTRIA QUÍMICA - SIQUIM}

O SIQUIM, localizado na Escola de Química de Universidade Federal do Rio de Janeiro, é um observatório de tendências de setores industriais, com atividades de monitoramento e prospecção, objetivando apoiar a tomada de decisão dos atores representantes do governo, da academia e das empresas.

Dentre as áreas de atuação de estudos e teses desenvolvidos no SIQUIM, tem-se a Gestão do Conhecimento; Prospecção Tecnológica e Mercadológica e, Inteligência Competitiva.

Estas três áreas apóiam-se em Sistemas de Informações e, tendo em vista que a indústria química se relaciona com praticamente todos os setores da economia, o SIQUIM é constituído de um conjunto de bases e de estudos de setores e segmentos, abrangendo:

análise tecnológica;

análise mercadológica;

usos mais relevantes;

novas tendências;

novos usos/diversificação em termos de ampliação;

levantamento sobre unidades produtoras ou futuras produtoras internacionais;

dados sobre mercado internacional;

capacitação nacional;

verticalização - insumos necessários para a produção de intermediários;

integração de indústrias - base e ponta;

identificação de lacunas e nichos na cadeia produtiva;

localização de produtores e disponibilidade de insumos;

tarifas aduaneiras;

identificação de novas moléculas;

árvore química;

levantamento de formulação de composição sinérgica;

prospecção tecnológica;

estudos sobre comércio exterior;

estudos dos setores industriais da economia;

- impacto do complexo químico na economia.

As técnicas de gestão do conhecimento, inteligência competitiva e prospecção tecnológica dão suporte às metodologias empregadas para os estudos de tendências setoriais, as quais usam ferramentas de tratamento da informação tendo como resultado mapas de conhecimento, para os tomadores de decisão.

\section{HISTÓRICO DO SIQUIM NA REALIZAÇÃO DE EVENTOS}

No ano de 1997, o SIQUIM, com apoio da FINEP/MCT/FUJB, realizou o "Seminário Internacional de Aprendizagem Tecnológica, Inovação e Políticas Industriais: Experiências Nacionais e Internacionais", em Angra dos Reis (RJ), contando com especialistas da área, tanto do país como da América Latina (Venezuela, Argentina, México e Cuba, com parceria com CENDES/ Universidad Central da Venezuela).

Já em 1998, na Escola de Química, foi organizado o workshop "Estudo prospectivo em Ciência e Tecnologia no Brasil", com a participação do Prof. Ron Johnston, Diretor Executivo do "Australian Center for Innovation \& International Competitiveness (ACIIC)", da Universidade de Sydney (Austrália).

Em 2000, na cidade de São Paulo, foi realizado o workshop
"Identificação dos Gargalos Tecnológicos Determinantes da Importação de Produtos Químicos", a fim de discutir os resultados obtidos no estudo, de mesmo nome, patrocinado pelo MCT PADCT - Plataforma Tecnológica em parceria com a ABIQUIM, que teve como objetivo central analisar, do ponto de vista tecnológico e industrial, os itens de maior relevância na pauta de importações de produtos químicos do País que apresentassem valor de importação superior a US\$ 10 milhões/ano.

Em 2001, foi realizado em São Paulo, com o apoio da FINEP, o "Workshop do Setor Químico-Farmacêutico: Futuros Genéricos", que contou com a participação de representantes da academia, empresas e governo, e teve como objetivo a definição do foco de estudo de projeto piloto sobre o setor químico farmacêutico, realizado em parceria com o Núcleo de Informação Tecnológica em Materiais, da UFSCar/DEMa.

O projeto "Identificação e Conhecimento da Balança Comercial do Complexo Químico", resultante de demanda realizada pela FINEP, foi realizado em 2002, envolvendo o estudo de todos os capítulos da Nomenclatura Comum do Mercosul (NCM) referentes ao complexo químico (27 ao 40) e identificação de produtos estratégicos na pauta de importação e exportação do País, em relação aos 4 mais importantes blocos econômicos que impactam o complexo químico (Nafta, União Européia, Mercosul e Tigres Asiáticos). Ao final do projeto foi organizado pelo SIQUIM, em São Paulo, workshop com representantes do Governo, academia e empresas para validação e análise dos resultados.

Em 2005 foi organizado pelo MDIC e UNIDO o "Seminário Internacional Prospecção em C,T\&I - Perspectivas de Integração Ibero-americana". No âmbito do Programa de Prospectiva Tecnológica Industrial das instituições organizadoras, o SIQUIM apresentou estudo sobre o Futuro da Indústria de Transformados Plásticos, no caso específico das embalagens para alimentos.

\section{Estudos realizados pelo SIQUIM relacionados à indústria de base}

A título de visualização, a Figura 1 apresenta exemplos de estudos sobre Indústria de Base - caso da cadeia produtiva de Petróleo e Gás.

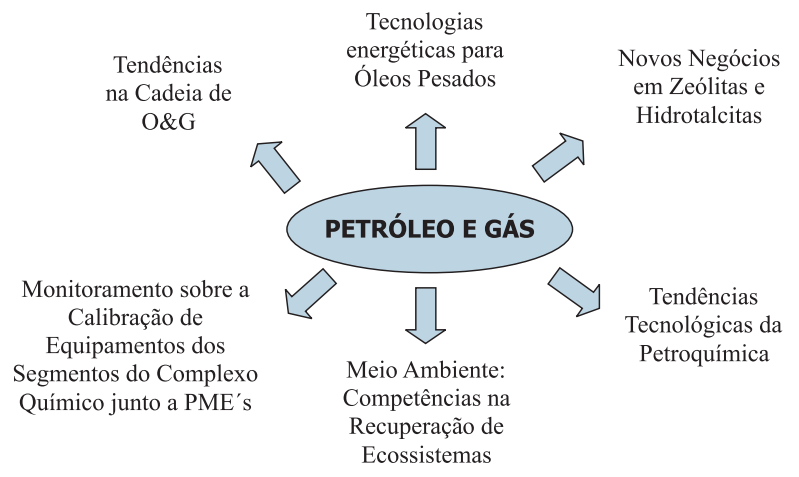

Figura 1. Estudos sobre a cadeia produtiva de petróleo e gás

A seguir, são apresentados pontos relevantes dos estudos relacionados na Figura 1.

Tendências na cadeia de petróleo e gás natural $(O \& G)^{I}$

As mudanças e impactos econômicos decorrentes do processo de globalização, bem como a política nacional de abertura da economia, no início da década de 90, fizeram-se sentir em vários seto- 
res e segmentos industriais do País. No que se refere mais especificamente ao setor $\mathrm{O} \& \mathrm{G}$, tais mudanças e novos requisitos vêm se confirmando como desafios a serem enfrentados e oportunidades a serem exploradas, principalmente após 1997, quando importantes medidas do governo sinalizaram uma nova etapa para este setor no Brasil (quebra do monopólio, "Lei do Petróleo - 9478" criando mecanismos de concessão do direito de exploração e produção e instituindo o CNPE e a ANP). Para tanto, esforços na direção tanto do entendimento das forças e movimentos deste setor em nível internacional e nacional, como na visualização de tendências tecnológicas e mercadológicas se fazem necessários, objetivando o estímulo à competitividade e à inovação do setor.

Neste contexto se insere este estudo, cujo objetivo foi levantar as inovações tecnológicas mais citadas em nível internacional de elos da cadeia produtiva de petróleo e gás (exploração \& produção, refino e gás natural - estocagem e transporte), no sentido de subsidiar a tomada de decisão.

A metodologia norteadora utilizou conceitos da Inteligência Competitiva e Prospectiva Tecnológica. Os levantamentos realizados para o período 2001-02 foram realizados com base em palavras-chave que nortearam a busca quanto à sua abrangência e precisão da área de interesse, nas seguintes fontes de informação:

- base de dados "Derwent- World Patent Index", com patentes indexadas pelo número de publicação; país; empresas ou entidades titulares; título e resumo da invenção;

- base de dados "Petroleum Abstracts", que fornece cobertura mundial de "papers", livros, artigos de jornais, teses, publicações de empresas, congressos e patentes em diversos assuntos da cadeia de petróleo e gás;

- anais dos dois últimos Congressos Internacionais de Petróleo (WPC), realizados em 2000 no Canadá e em 2002 no Brasil;

- informações provenientes da "World Wide Web" (WWW), contidas em robôs/máquinas de busca como www.teoma.com e www.copernicus.com.

Os resultados foram obtidos através do tratamento das informações em 3 níveis classificados como "macro", "meso" e "micro".

No nível "macro" foram gerados mapas de cada elo, provenientes das informações dos principais assuntos contidos nas patentes e artigos, identificando-se a quantidade de parcerias existentes (empresa/universidade/centro de pesquisa, empresa/empresa, empresa/associações); no nível "meso", foram identificadas as principais empresas detentoras de patentes, dentro de cada elo, identificando as principais tendências; já o nível "micro" apresenta os tópicos tecnológicos representativos das amostras estudadas (E\&P, Refino e Gás Natural), seguindo a especificidade de cada elo.

Como exemplo, mostra-se o resultado da pesquisa de patentes na base "Derwent" sobre estocagem e transporte de gás natural, visando acompanhar as tendências tecnológicas deste segmento na cadeia O\&G. O Japão se destaca com relação ao primeiro depósito, seguido dos Estados Unidos, conforme apresentado no Gráfico 1.

Com relação ao refino de petróleo, das 213 empresas/instituições depositantes, 4 se destacam no patenteamento, representando 26\% da amostra: China Petro-Chem (18 patentes); UOP LLC (16); ExxonMobil (11) e Institute Français du Petróle (10).

\section{Tecnologias energéticas para óleos pesados ${ }^{2}$}

Este estudo buscou construir visão macro, meso e micro sobre novas tecnologias utilizadas para processamento de petróleos pesados, como microondas, plasma, ultra-som e radiação ionizante/ ultravioleta, tendo como base a análise de conteúdo de patentes, artigos e anais de eventos. Além disso, foram mapeadas as competências no País.
Patentes Gás Natural - 2001/02

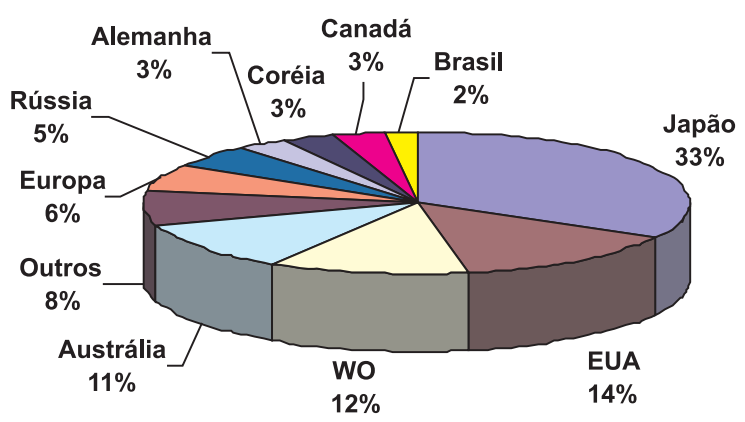

Fonte: Derwent.

Gráfico 1. Derwent: $1^{\circ}$ depósito de patentes em estocagem e transporte de gás natural

As fontes de informação pesquisadas e os respectivos resultados após validação foram:

- "Derwent World Patentes Index": base de dados de patentes, sendo analisados títulos, resumos, data de aplicação (prioridade) e depositantes das patentes referentes ao período 1993-03, nas seguintes tecnologias: microondas (154); plasma (61); ultrasom (7); radiação ionizante/ultravioleta (22).

- "Web of Science", base de artigos, período 1993-03, sendo analisados títulos, resumos e data de 32 artigos sobre microondas e de 29 sobre plasma.

- "Science Direct", base de artigos, período 1993-03, sendo analisados títulos, resumos e data de 127 artigos sobre plasma, não tendo sido identificados artigos sobre microondas.

- "Petroleum Abstracts", base de dados cobrindo artigos, eventos e patentes focados na cadeia de O\&G, no período 1993-03; foram resgatados 28 documentos sobre microondas.

- Sistema Lattes/CNPq, base de dados de currículos e grupos de pesquisa, para levantamento de especialistas em P\&D, com conhecimento nas áreas das energias de interesse do estudo. Foram identificados os seguintes números de especialistas por tecnologia: microondas (6); plasma (13); ultra-som (4); radiação ionizante/ultravioleta (7).

- Sistema SUPRIR (plataforma desenvolvida pelo CIN/CNEN que dissemina base de dados de eventos nacionais e internacionais). A pesquisa realizada para o período de 1993 a 2003 resultou em 29 eventos sobre microondas; 142 sobre plasma, 2 sobre ultra-som e 8 sobre radiação ionizante/ultravioleta.

\section{Novos negócios em zeólitas e hidrotalcitas ${ }^{3}$}

Alguns aluminossilicatos vêm sendo largamente utilizados em diversos processos industriais, como na catálise, adsorção, ou na troca iônica. Dois destes materiais, as zeólitas e as hidrotalcitas ${ }^{4-6}$ foram objeto deste estudo, que visou a Gestão do Conhecimento e Prospecção.

Para ambos os produtos, foram identificados, no período de 1993 a 2003, o número de patentes; sua origem; os depositantes mais expressivos; os mercados receptores de depósitos de patentes e, os principais conjuntos de classes técnicas que são objetos de patentes.

A Tabela 1, apresenta as 5 principais empresas depositantes em hidrotalcitas em 10 anos, e seus respectivos números de patentes.

No que concerne à competência brasileira em hidrotalcitas, foram levantados os pesquisadores e suas respectivas instituições, 
Tabela 1. Principais depositantes de patentes sobre hidrotalcita de 1993 a 2003

\begin{tabular}{lc}
\hline Depositantes (Corporação) & No $^{\circ}$ de Patentes \\
\hline Asahi Denka Kogyo KK & 70 \\
Kyowa Kagaku Kogyo KK & 32 \\
Sumitomo Bakelite Co. Ltd. & 30 \\
Mitsubishi & 27 \\
Henkel KGAA & 23 \\
\hline
\end{tabular}

titulações e e-mails; as instituições dominantes na pesquisa em termos de número de pesquisadores e, o quantitativo de pesquisadores por titulação acadêmica.

Para a competência brasileira em zeólitas, além dos mencionados para as hidrotalcitas, foram levantados mais 2 itens na base "Web of Science" no período 2000-03: os autores brasileiros com publicações; os principais autores brasileiros versus artigos publicados e, os artigos nacionais orientados a insumos e/ou aplicações relevantes versus grupos de pesquisa.

Foram também apontadas as tendências mercadológicas em zeólitas através dos movimentos de mercado no período de 2000/ 2002 e da Importação e Exportação do item genérico da Nomenclatura Comum Mercosul 3815.90.10 - Outros Iniciadores e Aceleradores para Coqueamento do Petróleo.

\section{Tendências tecnológicas da petroquímica}

Durante os últimos 20 anos, as tecnologias comercializadas no campo de petroquímicos foram focadas principalmente para poliolefinas. No entanto, há um número de inovações, muitas das quais podem ser chamadas de "nichos" tecnológicos, não significando fechamento de unidades ou mudança de rotas de produção, mas sim, adaptação para atender especificações "taylor-made".

As perspectivas tecnológicas vão ao encontro de atender o equilíbrio de oferta e demanda regional, utilizando mecanismos não tradicionais na petroquímica como otimização de matérias-primas consideradas co-produtos; outra força é a tecnologia como instrumento para gerar novos negócios, caso em que a biotecnologia e a nanotecnologia têm se mostrado promissoras, a primeira ambientalmente amigável e a segunda, por sua multidisciplinaridade e atendimento a mercados de valor agregado elevados, como o eletro-eletrônico.

As empresas integradas de petróleo/petroquímica estão investindo em plantas no mercado consumidor, preferindo esta opção a aumentar a capacidade no Oriente Médio e exportar o produto. Observa-se que das 25 maiores empresas de petróleo no mundo, 22 atuam na petroquímica.

Das empresas não integradas ao petróleo, 7 petroquímicas destacam-se mundialmente, algumas com unidades no Brasil: "Dow Chemical", "Basf", "BP Amoco", "DSM", "Exxon-Mobil", "Nova Chemical" e "Solvay". Estas apostam no mix de internacionalização, com atuação focada e global em produtos de suas "core competences". Há diversos casos de acordos, "joint ventures", fusões e aquisições.

$\mathrm{O}$ estudo focou as tecnologias relativas a produtos como olefinas/poliolefinas, p-xileno/PTA/PET, ácido acrílico/acrilatos, estireno e poliestireno (PS), além de fornecer uma visão de importação/exportação do Brasil destes produtos em 2003.

Dentre as principais tendências do século XXI identificadas, podem ser citadas a reciclagem, como parte integrante da estratégia de empresas que visam a manutenção de uma boa imagem frente à sociedade; o investimento em tecnologia para melhoria de processo e produtos e, o estabelecimento de alianças estratégicas en- tre empresas. Estas cooperações resultarão em maior concentração e conseqüente maior proteção de mercados e tecnologia, permitindo antecipar um cenário crítico de acesso às novas tecnologias para países tradicionalmente dependentes.

Meio ambiente: competências na recuperação de ecossistemas ${ }^{8}$

Este estudo teve como objetivo localizar as competências existentes no País aplicando a técnica de "text mining/data mining" a ecossistemas, utilizando tanto termos em português como em inglês, correlacionando-os à recuperação dos ecossistemas.

Para a busca de competências, foram utilizadas as seguintes fontes de informação: Plataforma Lattes/CNPq/MCT; "Web of Science" para o período de 1984 a 2004 (incluindo os artigos de autores estrangeiros que citam o Brasil) e, sites especializados, como a Base de Dados Tropical (BDT) e Restinga.net.

A Tabela 2 mostra, como ex., a distribuição dos 214 profissionais atuantes em restingas, localizados na base Restinga.net, por área de atuação.

Tabela 2. Base de dados Restinga.net - Distribuição de pesquisadores por área

\begin{tabular}{lc}
\hline Área de Concentração & $\mathrm{N}^{\circ}$ de Pesquisadores \\
\hline Botânica & 96 \\
Ecologia & 64 \\
Conservação & 21 \\
Zoologia & 13 \\
Geociências & 5 \\
Outras & 15 \\
\hline Total & 214 \\
\hline
\end{tabular}

Já com relação aos grupos de pesquisa cadastrados no Sistema Lattes/CNPq, foi realizado cruzamento dos termos de busca, tendo sido localizados 15 grupos, distribuídos nas seguintes áreas: Recursos Florestais e Engenharia Florestal (7); Ecologia (4); Oceanografia (2); Agronomia (1) e Engenharia Química (1).

A cada dia, nota-se mais intensamente a conscientização da sociedade a respeito das questões ambientais, e empresas, em especial as do setor de petróleo e gás, passam a ter como parte integrante de suas estratégias a preocupação com o meio ambiente. Neste sentido, a identificação e sistematização das competências nacionais tornam-se importantes no estabelecimento de parcerias para desenvolvimento de tecnologias voltadas à recuperação ambiental de ecossistemas específicos.

Monitoramento sobre a calibração de equipamentos dos segmentos do complexo químico junto a pequenas e médias empresas (PME's) ${ }^{9}$

Foi realizada pesquisa de campo, cujo instrumento de coleta de dados utilizado foi um questionário contendo perguntas abertas e fechadas sobre a demanda de ensaios e calibrações.

As 89 empresas entrevistadas localizam-se em 9 diferentes segmentos: Química Inorgânica; Química Orgânica; Resinas; Farmacêutico; Defensivos Agrícolas; Sabão, Limpeza e Perfumaria; Diversos; Borracha e, Plásticos, sendo predominantemente micro e pequenas empresas.

No que concerne à contratação de serviços metrológicos, 9 
empresas desconhecem fornecedores, 5 citam não o fazer por motivo do custo do serviço, 4 alegam que não há fornecedores para os serviços demandados e 1 empresa afirma que os prazos são a causa para não contratação.

Considerando o universo de empresas, apenas 5 instrumentos são presentes em mais de 1/3 das empresas da amostra: balança, paquímetro, termômetro, manômetro e micrômetro.

\section{Estudos realizados pelo SIQUIM com relação à indústria de ponta}

A Figura 2 refere-se aos estudos relacionados à Indústria de Ponta, caso exemplificado pela cadeia produtiva de Fármacos e Medicamentos:

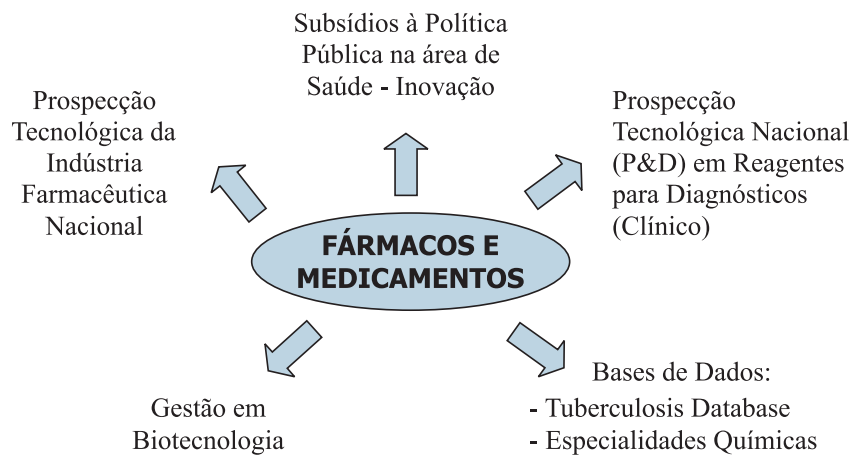

Figura 2. Estudos sobre a cadeia produtiva de Fármacos e Medicamentos

A seguir são apresentados alguns pontos relevantes dos estudos relacionados na Figura 2, ilustrando o monitoramento prospectivo.

\section{Prospecção tecnológica da indústria farmacêutica nacional ${ }^{10}$}

A Fundação Oswaldo Cruz (FIOCRUZ) lançou o projeto Inovação em Saúde, que visa, principalmente, fornecer subsídios para formulação de uma política multissetorial, envolvendo a gestão, o desenvolvimento científico e tecnológico e a produção de insumos críticos para a saúde. Para tanto, solicitou a realização de estudo sobre medicamentos.

O setor farmoquímico caracteriza-se, globalmente, pelo padrão de competição na diferenciação de produtos, e, mesmo sendo um oligopólio, metade do mercado mundial é dominado por alguns poucos líderes e, em determinadas classes terapêuticas, poucas empresas respondem por mais de $80 \%$ da demanda. Além disso, os investimentos em P\&D em medicamentos são extremamente elevados, e o desenvolvimento de novos medicamentos é tanto de alto custo como de alto risco, pois apenas 1 de cada 10 mil estudados é aprovado e chega à sociedade.

O estudo teve os seguintes objetivos:

- identificação dos medicamentos da Relação Nacional de Medicamentos - RENAME -com importância para a área de saúde pública (Prospecção Tecnológica e Mercadológica das drogas por Classes Terapêuticas, dos Medicamentos de Alto Custo e dos Genéricos da RENAME) e,

- mapeamento das competências do País para desenvolvimento de fármacos e medicamentos da RENAME (Líderes de Pesquisa, Rotas de Produção das drogas estratégicas da RENAME e Identificação das vias de Produção dos Fármacos).

Tendo em vista o grande número de variáveis correlacionadas, foram geradas 10 planilhas, que serviram de suporte para a identi- ficação de 88 produtos estratégicos: 3 delas referentes a fármacos (de Alta Importação e com Produção Nacional; de Alta Importação e sem Produção Nacional; e Superavitários), 3 referentes a medicamentos (de Alta Importação e com Produção Nacional; de Alta Importação e sem Produção Nacional do Fármaco; e Superavitários), e 4 relacionadas tanto a fármacos quanto a medicamentos (Produtos de Alta Importação de Fármaco e Medicamento; Produtos Estratégicos sem Produção de Genéricos no País; Produtos com Alto Potencial de Inovação; Medicamentos de Alto Custo).

Com base nas análises realizadas, foram também apresentadas recomendações de ações vis-à-vis a Política Industrial do País para Fármacos e Medicamentos, chegando a 15 produtos de interesse imediato.

Este estudo observou que há espaço para o desenvolvimento de fármacos em diferentes classes terapêuticas em termos de inovação, o que é demonstrado pelo patenteamento internacional e no País, porém, recomendam-se estudos que contemplem fármacos que extrapolem a RENAME. Foi identificada uma gama considerável de competências no País em várias etapas da cadeia produtiva de fármacos/medicamentos; verificou-se que, considerando a capacidade produtiva do País, é possível a substituição de importação competitiva e aumento das exportações, mas faltam incentivos fiscais necessários para a $\mathrm{P} \& \mathrm{D}$ nas indústrias. Desta forma, para levantar oportunidades de investimento visando a dinâmica de inovação, faz-se necessário o monitoramento sistemático de informação como subsídio aos atores envolvidos (governantes, empresas e pesquisadores).

\section{Subsídios à política pública na área de saúde - inovação ${ }^{11}$}

O objetivo principal deste estudo foi identificar a dinâmica de Pesquisa, Desenvolvimento e Inovação (P, D\&I) do setor saúde no cenário internacional, especificamente em relação a 3 categorias de doenças: crônicas (cardiovasculares, diabetes, e câncer), infectocontagiosas e negligenciadas (malária, leishmaniose e tuberculose), nos anos de 2001 e 2002.

Neste sentido, a Visão Internacional sobre Artigos Científicos foi obtida através da pesquisa na base de dados MEDLINE, identificando-se a ocorrência de artigos sobre as doenças-foco do estudo e sobre os medicamentos adotados pela RENAME, no período de 2001 e 2002. Além do total de artigos por doença, foi também identificado o número de "papers" relativos às categorias diagnóstico, terapia e prevenção e controle (utilizando-se para tanto o recurso da indexação do artigo por descritor).

Os artigos foram tratados para facilitar a identificação dos países líderes na produção técnico-científica por categoria, por doença, com as respectivas listas de termos técnicos, substâncias e instituições/empresas/autores. A partir dos resultados obtidos para cada categoria (terapia, diagnóstico, e prevenção), por doença, foram gerados mapas de conhecimento relativos aos termos técnicos de maior ocorrência, e mapas que correlacionam estes termos "top" com as 10 substâncias de maior freqüência, levando à sinalização das mais recentes pesquisas no mundo.

A Visão Internacional sobre Patentes foi obtida através de pesquisa na base de dados "Derwent World Patents Index". Para cada categoria (terapia, diagnóstico, e prevenção) por doença foi selecionada aquela de maior número de depósitos em 2001/2002. A partir da identificação da categoria líder em número de patentes por doença, foram identificadas as "top" empresas e suas tendências de patenteamento.

Para o caso de diabetes, mostrado aqui como ex., foram localizadas no período estudado 1890 patentes. Destacam-se aquelas 561 que envolvem as três categorias juntas, ou seja, tanto diagnóstico, 
como tratamento e também prevenção, com aproximadamente $29 \%$ do total. Deste sub-grupo de 561 patentes, a principal empresa depositante é a Incyte Genomics, com 90, cujas patentes abrangem, de maneira geral, não apenas diabetes, mas outros males como AIDS, câncer e infarto, e trazem como termos importantes os polipeptídeos e os polinucleotídeos.

Já a Visão Nacional sobre Pesquisadores e Empresas foi realizada utilizando-se o Sistema LATTES/CNPq, para localização de profissionais cujas áreas de atuação apresentam terminologias relacionadas à biotecnologia, verificando-se interface com o setor saúde; e diversos diretórios de Empresas de Biotecnologia, como os da BIOMINAS, BIORIO, e ABRABI.

No Gráfico 2 é apresentada a distribuição percentual, por Estado, das 83 empresas biotecnológicas atuantes na área de saúde localizadas no estudo, destacando-se Minas Gerais e Rio de Janeiro.

\section{Distribuição de empresas por Estado}

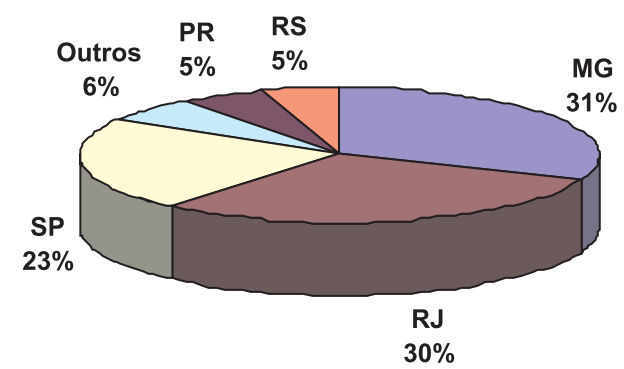

Gráfico 2. Distribuição por estado das empresas biotecnológicas atuantes em saúde

Prospecção tecnológica nacional $(P \& D)$ em reagentes diagnósticos (clínicos) ${ }^{12}$

No escopo do projeto Inovação em Saúde, objetivando fornecer subsídios para formulação de uma política multissetorial, e a produção de insumos críticos para a saúde, a FIOCRUZ solicitou a realização do estudo "Prospecção Tecnológica Nacional (P\&D) em Reagentes para Diagnósticos (clínicos) no âmbito da Saúde Pública", para detectar oportunidades de desenvolvimento de reagentes de diagnósticos laboratoriais que atendam às doenças transmissíveis/ controle de sangue: AIDS; Citomegalovírus; Dengue; Doença de Chagas; Filariose; Hepatites Virais; Herpes; HTLV I; HTLV II; Leishmaniose; Leptospirose; Malária; Rubéola; Sarampo; Sífilis e, Toxoplasmose.

Para o alcance do objetivo, foram elaboradas três diferentes visões: a de mercado, a de desenvolvimento e a de inovação.

Com relação à visão de mercado, praticamente este é dominado no País por importações, cujas informações encontram-se em 4 itens genéricos da Nomenclatura Comum Mercosul (3822.00.00 Reagente de Diagnóstico/Laboratório; 3822.00.10 - Reagente Determinante de Componente Sangue/Urina; 3002.90.10 Reagentes de origem microbiana para diagnóstico e, 3006.30.29 Outros reagentes de diagnóstico concebidos para serem administrados ao paciente), que exigiram abertura por guia de importação junto à Receita Federal, e como resultado pode ser ressaltada a concentração de poucas e relevantes empresas presentes neste mercado considerando-se valores em dólar (FOB).

Em relação à visão de desenvolvimento, a Academia (via currículo Lattes e indicação de especialistas) apresenta competência para atuação na área com 5 pesquisadores que confirmaram sua atuação em pesquisa básica e aplicada e 2 em pesquisa básica. Já o parque produtivo no país é incipiente, ainda que tenha sido localizado na pesquisa de campo, em algumas empresas, interesse na produção.

Em relação à visão de inovação na pesquisa de 2003/2004, as patentes internacionais analisadas tendem a serem amplas tanto em escopo (nas doenças) como em abrangência (se são de diagnóstico, tratamento e/ou prevenção). Foi possível observar que Hepatite e AIDS apresentam uma maior tendência de estarem na mesma patente. Além disso, ambas as doenças apresentam tendência de uso da biotecnologia para vacinas e diagnósticos.

Como conclusões, observam-se competências no País, inovação em termos de patentes no mundo, mas desorganização das informações de mercado no Brasil e, a princípio, desinteresse por parte das grandes empresas na produção de reagentes para identificação das doenças alvo do estudo.

\section{Gestão em Biotecnologia}

Trata-se da elaboração de um livro, organizado a partir de dissertações de mestrado e teses de doutorado orientadas pelos Profs. Adelaide Antunes e Nei Pereira Junior na Escola de Química da UFRJ, que será composto dos seguintes capítulos:

- O perfil da Biotecnologia no Brasil: Investimentos, Recursos Humanos e a Indústria de Biotecnologia.

- O Patenteamento em Biotecnologia

- Estratégias de Prospecção em Biotecnologia: O uso de drogas contra o câncer de mama

- A Biodiversidade Brasileira: O Caso da Amazônia

- Organismos Geneticamente Modificados: Uma Visão Estratégica

- A Influência Governamental na Indústria Nacional Farmoquímica e de Medicamentos

- Desenvolvimento Ambientalmente Sustentável

- Estratégia para a Ecoeficiência Aplicada às Águas: Produção mais Limpa

Com este livro, pretende-se ampliar a visão sistêmica aos cursos de Pós-graduação, no caso específico relacionado à Biotecnologia.

\section{Bases de Dados}

No ano de 2002, foram lançadas 2 bases de dados pelo SIQUIM, cujas principais informações são:

\section{TUBERCULOSIS DATABASE $^{13}$}

Base de dados formada por 74 drogas utilizadas no diagnóstico, prevenção e/ou combate à tuberculose, contendo as seguintes informações: CAS RN (Chemical Abstract Service Registry Number); Peso molecular; Fórmula molecular; Estrutura; Sinônimos; Nomes comerciais (com empresa produtora e país de origem); Atividade farmacológica; Aplicações terapêuticas; Rotas de produção; Combinações com outras drogas e Patentes relacionadas à droga desde 1962 (número, prioridade, data e depositante).

A busca no Cd-rom pode ser realizada através de palavras-chave, por produto, ou então por patente. A interface com o usuário é ilustrada na Figura 3.

\section{ESPECIALIDADES QUÍMICAS $^{14}$}

O Cd-rom "Especialidades Químicas" é composto por aproximadamente 250 produtos, tendo sido originado em um projeto de identificação de produtos classificados como de química fina ou "pseudo-commodities" com alta demanda nacional, que se consti- 


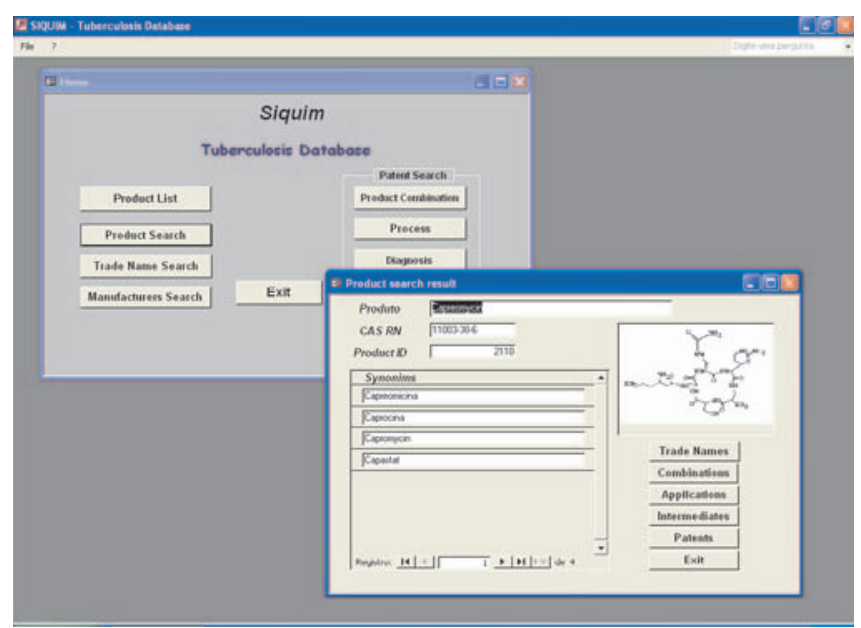

Figura 3. Tuberculosis Database

tuiriam em potenciais de investimento para a Indústria Química.

Para cada produto, estão disponíveis informações como: Nome químico; CAS RN (Chemical Abstract Service Registry Number); Classe química; Nomes comerciais; Fórmula estrutural; Principais insumos; Setores de aplicação; Empresas produtoras (nacionais e internacionais); Ex-produtores nacionais; NBM (Nomenclatura Brasileira de Mercadorias) e, NCM (Nomenclatura Comum do Mercosul).

Também é apresentada uma série histórica de importação desde 1999, mostrando casos de produtos classificados em NCMs genéricos. Por ano, tem-se a quantidade importada (em $\mathrm{kg}$ ); valor total (em US\$ FOB); e preço específico (em US\$ FOB/Kg).

Como exemplo, a Figura 4 apresenta alguns dados referentes ao diclofenaco sódico.
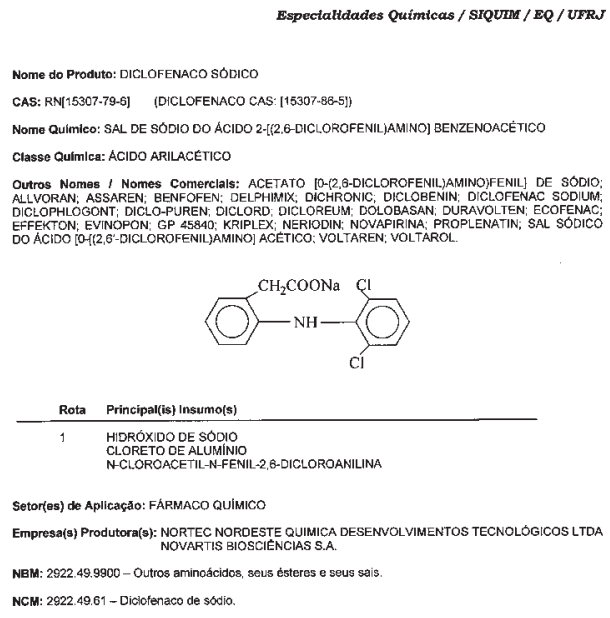

Figura 4. Especialidades Químicas - Diclofenaco sódico

O difícil acesso a informações de mercado, especialmente aos dados estatísticos de importação, é um entrave à visualização de novas oportunidades de investimento. Esta situação conflita com os objetivos do governo de aumentar a riqueza e as oportunidades de emprego, além de melhorar o desempenho da balança comercial do País.

Desta forma, este estudo torna-se importante ferramenta de trabalho para os tomadores de decisão, sejam do setor empresarial, acadêmico ou governamental.

\section{CONSIDERAÇÕES FINAIS/CONCLUSÕES}

No Brasil, onde o conhecimento está fortemente localizado nas Universidades e Centros de P\&D, é necessário que estes sejam o elo de ligação com o setor produtivo, no sentido de serem agentes da inovação para o alcance da sustentabilidade da competitividade.

Desta forma, o Observatório de Atividades Industriais e de Tendências em Ciência, Tecnologia e Inovação surge como modelo facilitador entre as Universidades, o Setor Produtivo e o Poder Público, visando a identificação de demandas geradoras de emprego e renda, através da informação e do conhecimento, das tendências sobre os impactos da tecnologia tanto na sociedade como na indústria.

O presente trabalho demonstra, por estudos realizados para o governo e empresas brasileiras, a prospecção de tendências tecnológicas e mercadológicas em setores de base e de ponta, apontando gargalos e alternativas de oportunidades para superação dos mesmos através da realização sistemática do acompanhamento de atividades industriais e do conhecimento, pela participação interativa dos especialistas.

\section{REFERÊNCIAS}

1. INT/Projeto Tendências; SIQUIM/EQ-UFRJ; Nota Técnica 03/2003: Mapeamento Tecnológico - Tendências Internacionais da Cadeia de O\&G: Exploração, Produção, Refino e Gás Natural, 2003; Disponível em http:// www.tendencias.int.gov.br/

2. SIQUIM/EQ-UFRJ; Gestão do Conhecimento e Prospecção em Processos Não Convencionais de Conversão de Óleos Pesados, 2004, Vols. 1 e 2.

3. SIQUIM/EQ-UFRJ; Gestão do Conhecimento e Prospecção em Zeólitas e Hidrotalcitas, 2003, vols. I-III.

4. Escobar, F. A. M.; Batista, M. S.; Urquieta-Gonzalez, E. A.U.; Quim. Nova 2000, 23, 303

5. Aguiar, M. R. M. P.; Novaes, A. C.; Guarino, A. W. S.; Quim. Nova 2002, 25,1145 .

6. Crepaldi, E. L.; Valim, J. B.; Quim. Nova 1998, 21, 300.

7. SIQUIM/EQ-UFRJ; Tendências Tecnológicas da petroquímica, 2004.

8. SIQUIM/EQ-UFRJ; Data mining em recuperação ambiental, 2004.

9. SIQUIM/EQ-UFRJ; Rede de Tecnologia/SEBRAE-RJ; Caracterização da demanda de serviços de ensaio e calibração, 2002.

10. Projeto Inovação em Saúde; SIQUIM/EQ-UFRJ; Prospecção tecnológica da indústria farmacêutica nacional, 2004, vol. I, II (A a F) e III.

11. CGEE e SIQUIM/EQ-UFRJ; Subsídios à Política Pública na área de Saúde - Inovação: Mapas do Conhecimento sobre tendências internacionais e competências nacionais em doenças crônicas, doenças infecto-contagiosas e doenças negligenciadas, 2003, vols. I, II e III. Disponível em: http:// www.mct.gov.br/Fontes/Fundos/cts/ctsaude/ CT_Saude_Estudos.htm

12. Projeto Inovação em Saúde; SIQUIM/EQ-UFRJ; Prospecção Tecnológica Nacional $(P \& D)$ em Reagentes para Diagnósticos (clínicos) no âmbito da Saúde Pública, 2005.

13. SIQUIM/EQ-UFRJ; Tuberculosis Database, Universidade Federal do Rio de Janeiro, Brasil, 2002.

14. SIQUIM/EQ-UFRJ; Alquimista Impaciente - Especialidades Químicas, Universidade Federal do Rio de Janeiro, Brasil, 2002. 